\title{
Modelo simulador de baixo custo para treinamento de septoplastia
}

\author{
Low cost simulator model for septoplasty training \\ Modelo simulador de bajo costo para entrenamiento en septoplastia
}

Felipe Xavier de Souza ${ }^{1 *}$, Jussandra Cardoso Rodrigues'1, Jocyane de Souza Andrade', Cassia Maria Oliveira de Oliveira', Ana Larisse Gondim Barbosa', André dos Santos Brandão'.

\section{RESUMO}

Objetivo: Desenvolver um modelo simulador de baixo custo para treinamento de técnicas de Septoplastia para médicos residentes em Otorrinolaringologia. Métodos: Trata-se de uma pesquisa tecnológica com desenvolvimento experimental de um protótipo de cirurgia. O modelo foi constituído por um septo artificial produzido com materiais de baixo custo e fácil aquisição, associado à um suporte em formato de hemi-crânio desenvolvido e impresso em impressora 3D. Adaptações foram realizadas para garantir que o resultado final fosse o mais objetivo e prático possível, facilitando assim sua utilização. Resultados: Com o modelo, foi possível treinar as etapas iniciais de um procedimento de septoplastia, podendo ser realizado em variados ambientes, devido seu baixo peso, facilitando seu transporte. Permite que o usuário se familiarize com os instrumentais de septoplastia e o uso de fotoforo em um espaço cirúrgico limitado que é a cavidade nasal, dando-lhes a oportunidade de melhorar a destreza manual, essencial durante um procedimento real. Conclusão: $O$ presente protótipo, oferece uma nova opção para treinamento e para melhorar as habilidades cirúrgicas do médico residente, conferindo maior segurança e destreza, mediante treinamento prévio em um modelo específico, para posterior transferência destas habilidades para um ambiente cirúrgico real.

Palavras-Chave: Otorrinolaringologia, Treinamento por simulação, Tecnologia de baixo custo.

\begin{abstract}
Objective: To develop a low cost simulator model for the training of Septoplasty techniques for physicians resident in Otorhinolaryngology. Methods: This is a technological research with experimental development of a surgery prototype. The model consisted of an artificial septum produced with low-cost and easy-to-acquire materials, associated with a hemi-skull shaped support developed and printed on a 3D printer. Adaptations were made to ensure that the final result was as objective and practical as possible, thus facilitating its use. Results: With the model, it was possible to train the initial stages of a septoplasty procedure, which can be performed in various environments, due to its low weight, facilitating its transport. It allows the user to become familiar with the septoplasty instruments and the use of photophorus in a limited surgical space that is the nasal cavity, giving them the opportunity to improve manual dexterity, essential during a real procedure. Conclusion: The present prototype offers a new option for training and to improve the surgical skills of the resident doctor, providing greater safety and dexterity, through prior training in a specific model, for later transfer of these skills to a real surgical environment.
\end{abstract}

Keywords: Otolaryngology, Simulation training, Low cost technology.

\section{RESUMEN}

Objetivo: Desarrollar un modelo de simulador de bajo costo para la capacitación de técnicas de septoplastia para médicos residentes en Otorrinolaringología. Métodos: Esta es una investigación tecnológica con

${ }^{1}$ Hospital Universitário Bettina Ferro de Souza, Belém-Pará. *E-mail: felipxavierSouza@gmail.com 
desarrollo experimental de un prototipo de cirugía. El modelo consistía en un tabique artificial producido con materiales de bajo costo y fáciles de adquirir, asociado con un soporte en forma de hemi-cráneo desarrollado e impreso en una impresora 3D. Se hicieron adaptaciones para asegurar que el resultado final fuera lo más objetivo y práctico posible, facilitando así su uso. Resultados: con el modelo, fue posible entrenar las etapas iniciales de un procedimiento de septoplastia, que puede realizarse en diversos entornos, debido a su bajo peso, lo que facilita su transporte. Permite al usuario familiarizarse con los instrumentos de septoplastia y el uso de fotóforo en un espacio quirúrgico limitado que es la cavidad nasal, lo que le brinda la oportunidad de mejorar la destreza manual, esencial durante un procedimiento real. Conclusión: El presente prototipo ofrece una nueva opción para el entrenamiento y para mejorar las habilidades quirúrgicas del médico residente, proporcionando mayor seguridad y destreza, a través del entrenamiento previo en un modelo específico, para la transferencia posterior de estas habilidades a un entorno quirúrgico real.

Palabras clave: Otolaringología, Entrenamiento simulado, Tecnología de bajo costo.

\section{INTRODUÇÃO}

A septoplastia é o terceiro procedimento cirúrgico mais comumente realizado por otorrinolaringologistas, após a miringotomia com inserção de tubo de ventilação e tonsilectomia com adenoidectomia (MANOUKIAN PD, et al., 1997). Nos Estados Unidos, a taxa anual de septoplastia foi de 8,7 por 10.000 habitantes em 2006, com aproximadamente 260.000 septoplastias realizadas a cada ano, compreendendo $43 \%$ das cirurgias nasosinusais (BHATTACHARYYA N, 2010).

No Brasil, em 2003, do total de 80.030 cirurgias otorrinolaringológicas, realizadas no âmbito do SUS, a septoplastia configura no terceiro procedimento cirúrgico mais realizado, após a tonsilectomia com adenoidectomia e redução cirúrgica de fraturas dos ossos próprios do nariz. Levando em consideração as cirurgias eletivas nasossinusais, a septoplastia foi a mais frequentemente realizada, seguido da rinoplastia e turbinectomia (GOUVEIA MCL, et al., 2003).

É um procedimento cirúrgico importante para correção do desvio do septo nasal, corrigindo assim quadros obstrutivos nasais e restabelecendo a anatomia e fisiologia adequada desta região. Além disso, ela fornece uma via de entrada em procedimentos rinológicos mais complexos, como rinoplastia e reconstrução septal, e pode ser necessário para melhorar o acesso como parte de um procedimento endoscópico (GUPTA S, et al., 2013). Várias são as técnicas utilizadas para septoplastia, levando em consideração os mais variados tipos e características de desvios septais, e preferências individuais do cirurgião (JIN HR e WON TB, 2008).

A grande maioria das técnicas clássicas de septoplastia são realizadas mediante a visualização das estruturas internas da cavidade nasal, por meio do uso de espéculos nasais de diferentes tamanhos, além do uso de fotóforo para a iluminação da anatomia septal, bem como sua relação com a complexidade anatômica desta região.

Segundo Alreefi MA (2017), ensinar a septoplastia aos médicos residentes pode ser difícil por várias razões, tais como: o tamanho relativamente pequeno das narinas; a dificuldade em observar os passos executados no interior da cavidade nasal; a anatomia complexa; os diversos padrões de desvio; risco de possíveis complicações, incluindo reparo inadequado, perfurações do septo nasal e vazamento de líquido cefalorraquidiano.

Desta forma, a curva de aprendizado dos residentes pode se tornar limitada, impondo dificuldades que poderiam ser corrigidas durante sua fase de treinamento. De acordo com os médicos residentes, a septoplastia é mal ensinada e não deve ser considerada como um procedimento simples (OAKLEY RL, et al., 2013; MARSHALL AH, et al., 2004).

Foi relatado que apenas $73,3 \%$ dos residentes em seu último ano de otorrinolaringologia se consideram adequadamente treinados para realizar o procedimento de forma independente (ALREEFI MA, et al., 2017), e os residentes expostos a instrumentos de treinamento em septoplastia relataram estarem mais bem preparados do que aqueles que não foram expostos a este artifício (GUPTA S, et al., 2013). 
Em uma recente revisão sistemática realizada por van Egmond MMHT, et al (2018), sobre o tratamento cirúrgico de desvio do septo nasal, as complicações mais frequentes observadas foram perfuração septal, sinéquias, hematoma septal e hemorragia secundária. Complicações essas que podem ser ainda mais frequentes quando o procedimento cirúrgico for realizado por médicos inexperientes ou residentes de otorrinolaringologia em treinamento.

O desenvolvimento de habilidades adequadas depende da prática deliberada e repetitiva, de tarefas apropriadas e de feedback imediato para correção de erros (ERICSSON K, 2004). Desta forma o desenvolvimento e aprimoramento de simuladores de treinamento cirúrgico representa uma importante alternativa na educação médico-cirúrgica, oferecendo um ambiente no qual os alunos e residentes possam treinar até atingir níveis específicos de competência, com benefício ao seu aprimoramento por meio de procedimentos repetitivos supervisionados, permitindo a detecção e a análise de erros cirúrgicos sem risco para o paciente (FRIED MP, 2010).

Os simuladores disponíveis atualmente nos grandes centros médicos-cirúrgicos variam de sofisticados manequins, modelos cirúrgicos impressos em impressoras 3D, cadáveres para dissecção, até dispositivos virtuais de treinamento por computador e realidade virtual (BARREIRA MA, et al., 2008). Modelos estes de custo elevado ou de difícil acesso, onde o valor de aquisição é um fator muito importante a ser analisado, já que essas tecnologias médicas não podem ser oferecidas em locais onde os recursos financeiros são limitados.

A prática da simulação contemporânea é relativamente recente e o interesse está crescendo, mas ainda não houve investimentos extensos na aquisição de simuladores para os programas de treinamento em otorrinolaringologia (JAVIA L e DEUTSCH ES, 2012). Espera-se que o aumento da demanda por simuladores no futuro gere a produção mais acessíveis desse importante instrumento de ensino prático. Desta forma, visando fornecer uma ferramenta de treinamento cirúrgico e de prática de novas habilidades, para os médicos residentes em formação no curso de otorrinolaringologia, este artigo teve como objetivo criar um protótipo inicial de treinamento, de baixo custo, de fácil produção e acesso, para a realização das etapas iniciais de procedimentos de septoplastia.

\section{MÉTODOS}

Trata-se de uma pesquisa tecnológica com desenvolvimento experimental de um protótipo de cirurgia, realizado na Cidade de Belém-Pará. Devido à natureza do projeto, nenhuma aprovação de Comitê de ética em pesquisa ou autorização de qualquer outra instituição foram necessárias. Com o interesse da construção de um modelo de treinamento para septoplastia de baixo custo e de fácil produção, buscou-se materiais baratos e de fácil acesso em nosso cotidiano para a sua confecção. O projeto foi estruturado em 3 etapas: idealização da pesquisa, estudo sobre a viabilidade do projeto e produção do protótipo de treinamento, totalizando um tempo de aproximadamente 11 meses para chegar ao resultado atual. Todo o custo da pesquisa ficou de responsabilidade do autor, não havendo financiamento de qualquer colaborador ou instituição financeira.

O primeiro passo foi a construção da estrutura rígida do septo nasal, formado por suas estruturas ósseocartilaginosas. Para produção das estruturas cartilaginosas, optou-se pelo uso dos recipientes de plástico de soro fisiológico nasal $0,9 \%$, mais precisamente sua porção central, por ser bem mais maleável e delicado que o restante do recipiente. Para realizar a demarcação e corte do recipiente utilizou-se um molde em folha de PVC com as devidas proporções da porção cartilaginosa do septo nasal.

O segundo passo foi a produção da porção óssea do septo nasal, formado pela porção perpendicular do osso etmoide e pelo Vômer. Foi utilizado uma lâmina de isopor com $40 \mathrm{~mm}$ de espessura, sendo cortado utilizando como base um molde, também em folha de Policloreto de Polivinila (PVC), na proporção de 1:1 do septo nasal. O plástico representativo da porção cartilaginosa do septo, foi encaixado na linha média da espessura do isopor. Após a exposição do conjunto a uma chapa de metal aquecida, este foi reduzido à uma espessura de $0,9 \mathrm{~mm}$, tornando-se mais rígido, assemelhando-se com a consistência e textura da estrutura óssea septal (Figura 1). 
Figura 1 - Conjunto plástico e isopor, após aquecimento com chapa de metal aquecida.

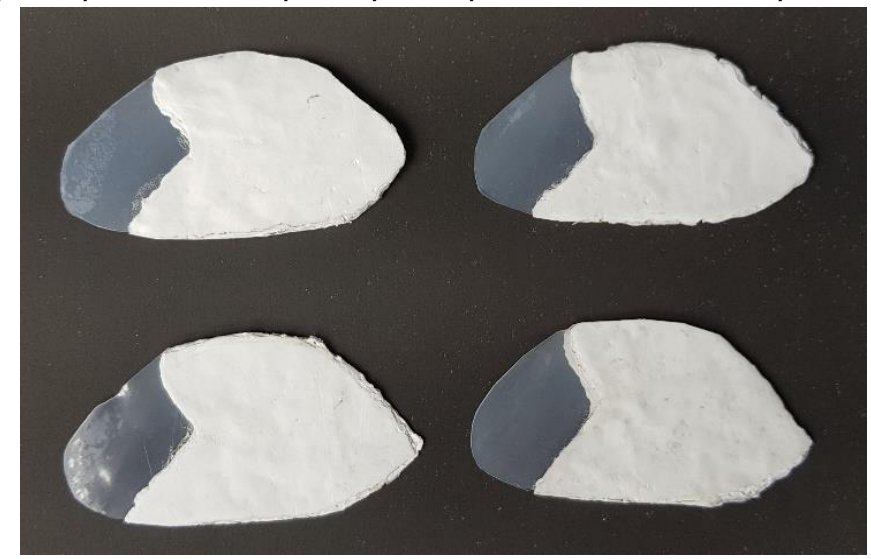

Fonte: Souza FX, et al., 2019.

Para compor o mucoperiósteo e mucopericôndrio, utilizou-se papel adesivo transparente tipo Contact, fixando sua parte adesiva em ambos os lados do modelo ósseo-cartilaginoso. Devido à dificuldade de acesso a látex, seu complexo manuseio e alto custo, optou-se pelo uso de folhas de pele sintética feitas de silicone com 1mm de espessura, para simular a mucosa septal. Esse material é muito utilizado para treinamento de pigmentação estética de sobrancelhas e tatuagens, de fácil acesso à internet. Este foi colado sobre o papel Contact mediante uso de cola adesiva instantânea multiuso TekBond® 200 , de alta viscosidade. $O$ modelo completo formado com todas os materiais utilizados possui cerca de $90 \mathrm{~mm}$ de comprimento por $48 \mathrm{~mm}$ de altura, além de espessura máxima de $5 \mathrm{~mm}$ (Figura 2).

Figura 2 - Estrutura final do modelo de septo nasal.

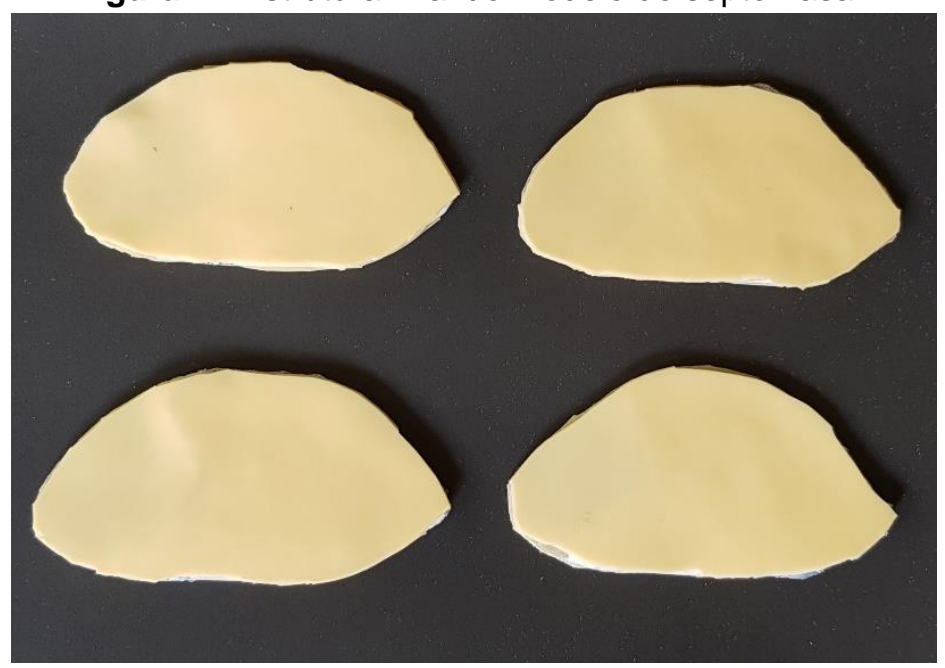

Fonte: Souza FX, et al., 2019.

Para servir de suporte ao septo artificial, foi desenvolvido com a ajuda de um prototipista de impressão 3D, um hemi-crânio, apenas com seu componente ósseo, na proporção de 1:1, como forma de servir de suporte ao modelo de septo artificial, além de simular a posição cirúrgica da cabeça humana com leve extensão cervical. A impressora utilizada no processo de criação do suporte foi a 3D PRO-GTMAX3D CORE A3, utilizando como matéria prima para confecção o filamento Acrilonitrila Butadieno Estireno (ABS) MG94. A cavidade nasal do suporte foi desenvolvida vazada, apresentando uma canaleta superior e uma inferior, que se encontram anteriormente e posteriormente, permitindo assim a passagem do septo artificial, garantindo sua fixação (Figura 3). O suporte 3D não possui os componentes externos do nariz, garantindo a ampla visualização do septo artificial. 
Figura 3 - Estrutura final do modelo de septoplastia integrado ao suporte impresso em Impressora 3D.

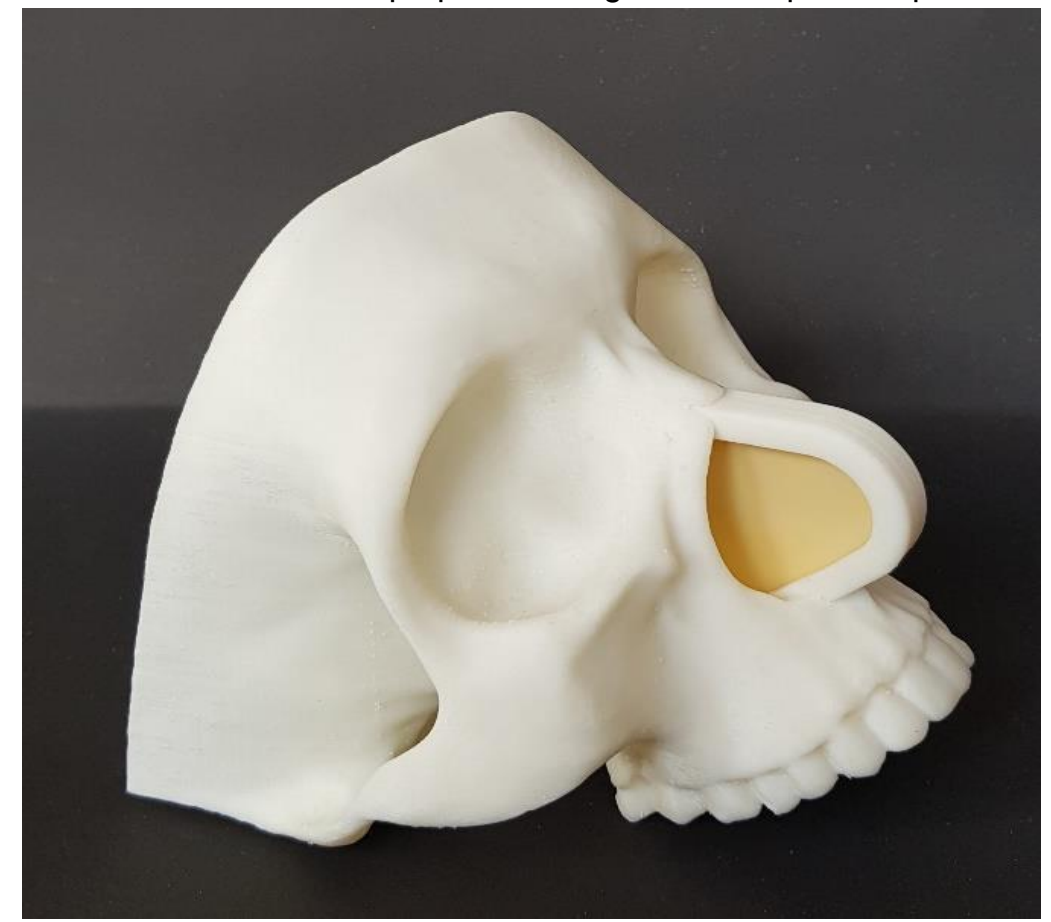

Fonte: Souza FX, et al., 2019.

O custo total dos materiais utilizados girou em torno de $R \$ 170,00$, levando em consideração o gasto zero com os recipientes de soro fisiológico, que muitas vezes são descartados. Foi possível a produção de aproximadamente 15 a 20 septo artificial, com um custo aproximado de $\mathrm{R} \$ 8,50$ a 11,50 , por modelo criado. Em relação ao suporte, o valor total foi de aproximadamente $R \$ 450,00$, levando em consideração o valor dos honorários do prototipista, do material utilizado para impressão do suporte e do custo do envio.

\section{RESULTADOS}

O modelo de treinamento criado representa um protótipo inicial, sendo previsto que alterações futuras sejam realizadas, para conferir ao produto final maior praticidade e funcionabilidade, mantendo o baixo custo de produção e manutenção. Garantindo assim, que vários treinamentos possam ser realizados, quantas vezes forem necessárias, após a simples substituição do septo artificial utilizado por um outro novo.

Com o modelo foi possível treinar as etapas iniciais de procedimento de septoplastia, podendo ser realizado de maneira sucessiva, após a limpeza simples do suporte, retirando qualquer resíduo da canaleta de fixação, para que um novo septo artificial pudesse ser encaixado. Para isso há a necessidade de produção prévia de número adequado de septos artificiais, de acordo com o número de procedimentos para treinamento que estejam planejados para o dia.

Por ser constituído totalmente de material sintético, sem trazer riscos de contaminação biológica, e por ser de baixo peso, facilitando seu transporte, pode ser reproduzido e utilizado em qualquer local, seja ambiente residencial ou hospitalar. Podem ser utilizados instrumentais cirúrgicos antigos e de qualidade reduzida para o treinamento, sem trazer prejuízo a prática do exercício no modelo criado.

O modelo também permite que os residentes se familiarizem com os instrumentais de septoplastia e o uso de fotoforo em um espaço cirúrgico limitado que é a cavidade nasal, dando-lhes a oportunidade de destreza manual e a habilidade essencial para um procedimento real (Figura 4). O treinamento em modelo sintético aqui proposto ainda não objetivou ensinar os residentes ao tratamento de alterações patológicas septais, pois todos os septos artificiais produzidos apresentam-se retificados, para garantir encaixe adequado na caneleta do protótipo. 
Figura 4 - Uso de instrumental cirúrgico auxiliado pela visualização sob luz de fotóforo.

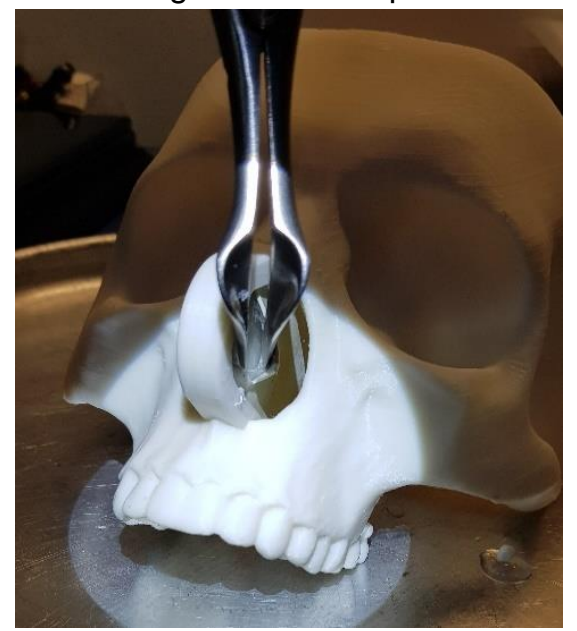

Fonte: Souza FX, et al., 2019.

A presença do suporte garantiu posicionamento adequado do septo artificial, proporcionando ao residente que suas duas mãos estivessem livres para a manipulação do instrumental cirúrgico. Foi possível realizar o treinamento básico e demonstração do passo-a-passo inicial de uma septoplastia utilizando-se a técnica de Killian, visto que a conformação das canaletas do suporte não permite a manipulação necessária da porção caudal do modelo do septo artificial, impedindo a realização das demais técnicas cirúrgicas.

Foi também possível observar a simulação da incisão da mucosa com a utilização de lâmina número 15 acoplada em cabo de bisturi, tendo a pele sintética de silicone demonstrado apresentar consistência semelhante a mucosa humana verdadeira; a obtenção e o adequado descolamento do plano submucopericondrial bilateralmente (Figura 5); realizar as condrotomia anterior e superior, mediante uso de lâmina número 15 e a condrotomia posterior através do descolamento da cartilagem representada pelo plástico da porção óssea constituído por isopor compacto; a ressecção da porção óssea por meio de tesoura reta; além de sutura da mucosa incisada utilizando-se fio catgut 3.0.

Figura 5 - Obtenção de plano abaixo ao papel contact, representativo do plano subpericondrial.

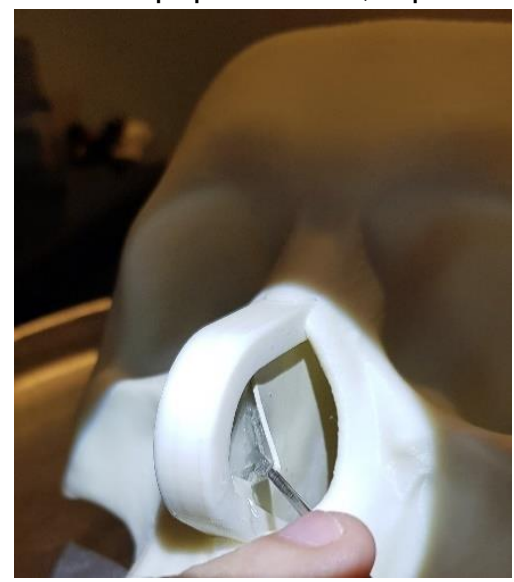

Fonte: Souza FX, et al., 2019.

Com o modelo pode-se realizar o treinamento de colocação e fixação de Splint de diferentes materiais e tamanhos (Figura 6). Além disso, permitiu a realização de pontos para retificação septal, seja eles efetivados anteriormente ou posteriormente. Após o término do treinamento, o septo artificial utilizado pode ser descartado em depósito apropriado para reciclagem, por ser constituído basicamente de silicone, plástico e isopor, sem trazer riscos de contaminação ao meio ambiente. 
Figura 6 - Splint de silicone posicionado e fixado ao septo artificial.

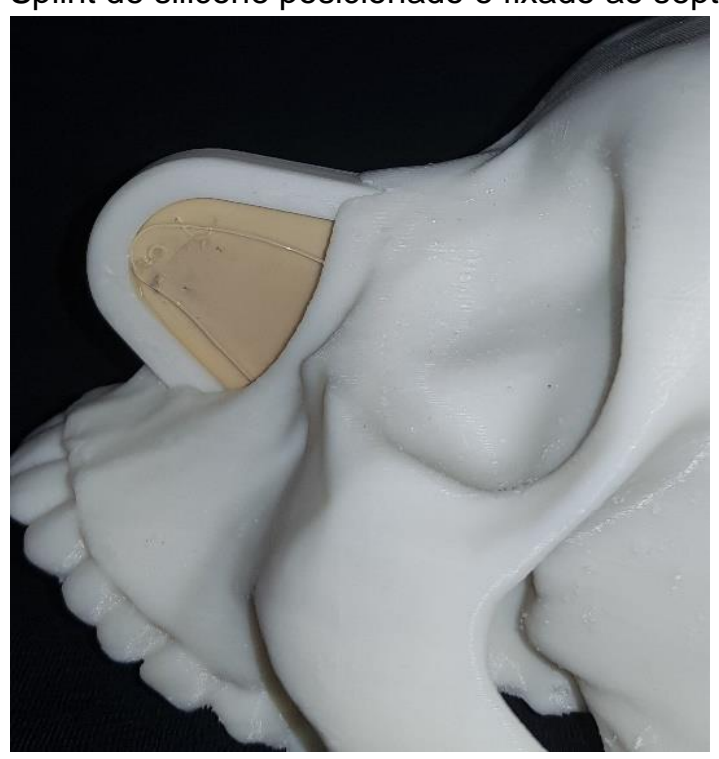

Fonte: Souza FX, et al., 2019.

\section{DISCUSSÃO}

O modelo de septoplastia produzido, integrado ao suporte formulado em impressão 3D, oferece aos residentes de otorrinolaringologia, uma clara oportunidade de treinamento em habilidades cirúrgicas. No atual cenário, as evidências disponíveis apontam que a prática baseada em simulação apresenta importância notável no aprendizado, resultando na transferência das habilidades adquiridas em modelos de treinamentos para o ambiente cirúrgico real (JAVIA L e DEUTSCH ES, 2012). Desta forma, o treinamento preciso e extenso é fundamental na formação do médico residente em otorrinolaringologia (OLIVEIRA HF, 2018).

O modelo criado é de baixo custo, composto por materiais simples e de fácil acesso, podendo ser produzido por qualquer pessoa após simples capacitação. É de baixo peso, e portátil, podendo ser reproduzido e utilizado em qualquer local, não limitando sua utilização em ambiente hospitalar. Apresenta muitas diferenças dos modelos sintéticos disponíveis atualmente, considerados de custo elevado, costumeiramente descartados após uma única manipulação (NOGUEIRA JF, et al., 2008; STAMM A, et al., 2009), sendo estes últimos considerados inviáveis economicamente para a maioria dos serviços públicos de residência médica, caso usados em frequência adequada.

O projeto passou por vários testes e modificações, para garantir que o protótipo inicial fosse o mais objetivo e prático possível, garantindo assim adequada otimização e fácil manuseio por parte dos residentes e preceptores, dada a simples tecnologia utilizada para sua confecção. Dentre as modificações realizadas no suporte 3D, está a maior projeção do septo nasal para exterior, garantido um melhor acesso e manipulação deste; além de uma cavidade nasal mais alargada, facilitando o manuseio dos instrumentais, devido à ausência de elasticidade do material que compõem o suporte feito por impressora 3D.

Isso facilitaria a boa observação por parte do corpo docente, que poderia realizar as instruções adequadas, devido a facilidade de visualização direta do que está sendo realizado pelo médico residente. Pois segundo Javia L e Deutsch ES (2012), isso permite que os estudantes pratiquem habilidades selecionadas, recebam feedback do corpo docente e continuem a praticar conforme necessário, com base nas necessidades educacionais do praticante, e não nas necessidades médicas do paciente, e permitam que essa prática ocorra sem risco direto a este.

A pele sintética de silicone utilizada para substituir a mucosa septal, apresentava textura semelhante a habitual, garantindo boa manipulação, tanto durante a incisão com bisturi, quanto durante a elevação do retalho. O manipulador deveria ter o cuidado para não realizar movimento intempestivos, que poderiam lacerar a pele artificial, assemelhando-se assim ao cuidado que normalmente deve ser realizado durante 0 
procedimento cirúrgico. Pois Fuchs J (1946), em meados do século XX, já entendia a importância de deixar a mucosa e o mucopericôndrio ligado à cartilagem septal de um lado para evitar perfurações septais pósoperatórias.

O papel adesivo transparente tipo Contact, com sua face adesiva voltada para o plástico e isopor, garantiu uma boa fixação com essas estruturas, impondo certa dificuldade para obtenção do seu plano aderente e de seu descolamento durante $o$ ato de treinamento. Dificuldade está semelhante ao observado na obtenção do plano do mucoperiósteo e mucopericôndrio durante uma septoplastia real, sendo um importante ponto de treinamento observado no modelo artificial. Pois segundo Garzaro M, et al (2019), o descolamento limitado inicial e o reposicionamento final da mucosa representam as fases mais cruciais para minimizar o dano da mucosa e, subsequentemente, reduzir o risco de desenvolver sinéquias.

Entretanto, o sítio que exigiu maior atenção e dificuldades, foi o plástico mimetizando a cartilagem septal. Por ser transparente e mais rígido que a cartilagem real, impôs importante dificuldade durante sua incisão e posterior condrotomias, exigindo que o operador usasse força, o que não era natural, tornando-se cansativo e não reproduzia a força necessária empregada em sala de cirurgia. Entretanto, garantiu pouca dificuldade na passagem da agulha de sutura, sendo importante assim para o treinamento de colocação e fixação de splint, e os diversos tipos de suturas e pontos que costumeiramente são realizados no ato cirúrgico para melhor retificação do septo nasal (KANG JM, et al., 2012).

Um quesito não satisfatório observado e que necessita ser aperfeiçoado, foi a impossibilidade de realizar a hidrodissecção, por provável maior rigidez dos materiais empregados na produção do modelo, dificultando a infusão de soro fisiológico 0,9\% mediante seringa de $10 \mathrm{ml}$ e jelco $\mathrm{n}^{\circ} \mathbf{1 5}$, havendo a necessidade de realizar força acentuada, sem a obtenção do resultado adequado. Achado semelhante foi encontrado no trabalho de Alreefi MA, et al (2016), que criou um modelo de nariz totalmente em impressora 3D, para treinamento de septoplastia, apresentando dificuldades na elevação do retalho mucoso por maior aderência e rigidez do material utilizado.

Outro ponto que precisa ser melhorado no modelo é que, usando os materiais disponíveis, o sangramento não pôde ser reproduzido, o que é uma parte importante do procedimento. Além disso, a pele artificial de silicone apresenta uma coloração diferente da coloração habitual da mucosa septal, e o material de plástico empregado para formar o septo cartilaginoso ser transparente, limitando a precisão da profundidade da incisão com o bisturi durante o treinamento, ocasionando maior risco de laceração contralateral.

Esse foi o primeiro passo para a construção de um modelo de treinamento para septoplastias, onde várias melhorias devem ainda ser realizadas. Dentre elas, a busca de novos materiais que possam substituir os usados atualmente e mimetizar o tecido humano com maior fidelidade e que ainda assim seja de baixo custo, para posteriormente padronizar seu processo de validação e obtenção do seu provável efeito na transferência das habilidades para a sala de cirurgia.

Pois segundo Anastakis DJ, et al (1999), o exercício de residentes em modelos de treinamento com maior fidelidade, permite a transferência das habilidades técnicas adquiridas para modelos humanos e, posteriormente, para o centro cirúrgico, tendo os simuladores, sido usados com sucesso para aquisição ou melhoria de habilidades (VAN EGMOND MMHT, et al., 2018), diminuindo a quantidade de treinamento necessária na sala de cirurgia (SCHAEFER JJ, 2004).

\section{CONSIDERAÇÕES FINAIS}

O modelo proposto permite o desdobramento em trabalho futuro de modificações no protótipo atual ao incluir a reprodução de desvios septais patológicos e introdução de estruturas anatômicas como cornetos nasais, para treinamento conjunto de turbinectomia inferior. Propõe-se ainda, após as devidas adaptações, a submissão do simulador à validação por parte dos residentes em Otorrinolaringologia a fim de documentar as competências e habilidades adquiridas no modelo e sua repercussão quando executado em sala de cirurgia com pacientes reais. Oferece uma opção barata, de fácil acesso e reprodução para a prática e melhor aquisição de habilidades cirúrgicas pelos médicos residentes em formação, frente a falta de recursos de ensino e dificuldades encontradas na atual situação da saúde pública hospitalar. 


\section{REFERÊNCIAS}

1. ALREEFI MA, et al. Development and validation of a septoplasty training model using 3-dimensional printing technology. Int Forum Allergy Rhinol, 2017; 7(4): 399-404.

2. ANASTAKIS DJ, et al. Assessment of technical skills transfer from the bench training model to the human model. Am J Surg, 1999; 177: 167-170.

3. BARREIRA MA, et al. Model for simulated training of surgical anastomoses by laparoscopy. Rev Med UFC, 2008; 58(3): 65-67.

4. BHATTACHARYYA N. Ambulatory sinus and nasal surgery in the United States: demo-graphics and perioperative outcomes. Laryngoscope, 2010; 120(3): 635-638.

5. ERICSSON K. Deliberate practice and the acquisition and maintenance of expert performance in medicine and related domains. Acad Med, 2004; 79(10): 70-81.

6. FRIED MP, et al. From virtual reality to the operating room: The endoscopic sinus surgery simulator experimente. Otolaryngol Head Neck Surg, 2010; 142(2): 202-207.

7. FUCHS J. A rapid approach to the deviated septum in rhinoplasty. Plast Reconstr Surg, 1946; 18: 133-140.

8. GARZARO M, et al. Endoscopic versus conventional septoplasty: objective/subjective data on 276 patients. Eur Arch Otorhinolaryngol, 2019; 276(6): 1707-1711.

9. GOUVEIA MCL, et al. Perfil de internamento por morbidade otorrinolaringológica com tratamento cirúrgico. Brasil, 2003. Revista Brasileira De Otorrinolaringologia, 2005; 71(6): 698-704.

10. GUPTA S, et al. Septoplasty trainin. Ann R Coll Surg Engl, 2013; 95: 10-13.

11. JAVIA L, DEUTSCH ES. A Systematic Review of Simulators in Otolaryngology. Otolaryngol Head Neck Surg, 2012; 147(6): 999-1011.

12. JIN HR, WON TB. Septoplasty: current concept and technique. J Rhinol, 2008; 15: 13-29.

13. KANG JM, et al. Modified mattress suturing technique for correcting the septal high dorsal deviation around the keystone area. Am J Rhinol Allergy, 2012; 26(3): 227-232.

14. MANOUKIAN PD, et al. Recent Trends in Utilization of Procedures in Otolaryngology-head and Neck Surgery. Laryngoscope, 1997; 107(4): 472-477.

15. MARSHALL AH, et al. Principles of septal correction. The Journal of Laryngology \& Otology, 2004; 118: $129-134$.

16. NOGUEIRA JF, et al. Building a real endoscopic sinus and skull-base surgery simulator. Otolaryngol Head Neck Surg, 2008; 139(5): 727-728.

17. OAKLEY RJ, et al. Septal surgery:were you trained? R Coll Surg Bull, 2013; 84: 15-17.

18. OLIVEIRA HF. Modelo de treinamento e aquisição de habilidades em cirurgias endoscópicas nasais em cabeça de cordeiro. Tese (Doutorado em Ciências Médicas) Universidade de Brasília, 2018; 49 p.

19. SCHAEFER JJ. Simulators and difficult airway management skills. Paediatr Anaesth, 2004; 14: 28-37.

20. STAMM A, et al. Feasibility of balloon dilatation in endoscopic sinus surgery simulator. Otolaryngol Head Neck Surg, 2009; 140(3): 320-323.

21. VAN EGMOND MMHT, et al. Septoplastia for nasal obstruction due to a deviated nasal septum in adults: a systematic review. Rhinology, 2018; 56(3): 195-208. 